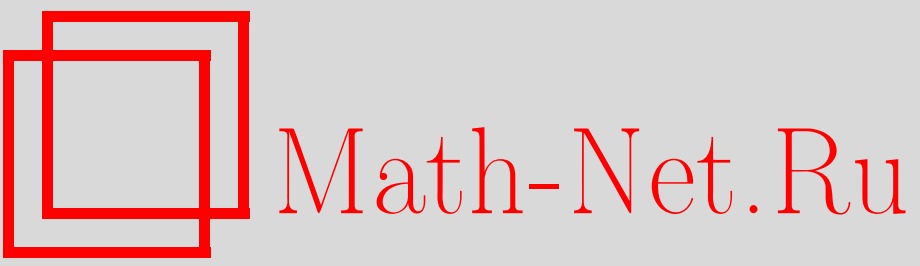

А. Ю. Захаров, И. К. Локтионов, Классическая статистика однокомпонентных систем с модельными потенциалами, ТМФ, 1999, том 119, номер 1, 167-176

DOI: https://doi.org/10.4213/tmf735

Использование Общероссийского математического портала Math-Net.Ru подразумевает, что вы прочитали и согласны с пользовательским соглашением

http://www.mathnet.ru/rus/agreement

Параметры загрузки:

IP : 54.210 .77 .194

26 апреля 2023 г., 16:01:42 
ТЕОРЕТИЧЕСКАЯ

И МАТЕМАТИЧЕСКАЯ

ФИЗИКА

Том 119, № 1

апрель, 1999

(C) 1999 г.

А.Ю. Захаров ${ }^{*}$ И.К. Локтионов ${ }^{\dagger}$

\section{КЛАССИЧЕСКАЯ СТАТИСТИКА ОДНОКОМПОНЕНТНЫХ СИСТЕМ \\ С МОДЕЛЬНЫМИ ПОТЕНЦИАЛАМИ}

Получено представление конфигурационного интеграла однокомпонентных классических систем с двухчастичными потенциалами, разложимыми в интеграл Фурье, в виде континуального интеграла. В данном представлении выполнена факторизация подынтегрального выражения по атомным координатам. "Одноатомные" сомножители носят универсальный (т.е. не зависящий от явного вида межатомных потенциалов) характер. Получено достаточное условие спонтанного нарушения симметрии в непрерывных моделях классической статистики. Исследован случай модельных потенциалов с неотрицательными фурье-образами. Для потенциалов этого класса континуальный интеграл вычислен методом перевала. Установлено существование фазовых переходов для некоторых модельных потенциалов.

\section{1. ВВЕДЕНИЕ}

Проблема фазовых переходов относится к числу наиболее сложных и важных в статистической механике. В обшей постановке эта проблема разбивается на две части. Первая - нахождение "реальных" межатомных взаимодействий. Вторая - расчет статистической суммы системы с известными взаимодействиями. Обе эти проблемы пока еше весьма далеки от решения.

В работе [1] предложен метод факторизации в классической статистике. Цель данной работы состоит в преобразовании конфигурационного интеграла

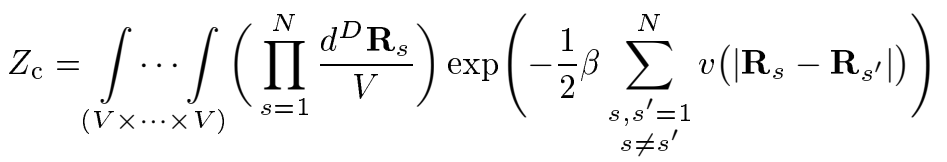

системы классических частиц для определенного класса межатомных потенциалов к виду, позволяюшему сделать определенные заключения о фазовых переходах, и в применении результата к описанию фазовых переходов.

\footnotetext{
* Новгородский государственный университет, Новгород, Россия. E-mail: theorphy@info.novsu.ac.ru

$\dagger$ Донецкий государственный технический университет, Донецк, Украина. E-mail: innego@public.donetsk.ua
} 


\section{2. КОНТИНУАЛЬНОЕ ПРЕДСТАВЛЕНИЕ КОНФИГУРАЦИОННОГО ИНТЕГРАЛА И ФАКТОРИЗАЦИЯ ПО АТОМНЫМ КООР ДИНАТАМ}

Будем предполагать, что $N$ частиц в классической системе объема $V$ взаимодействуют между собой через известный двухчастичный центральный потенциал $v(\mathbf{r})$, который допускает разложение Фурье

$$
v(\mathbf{r})=\frac{1}{V} \sum_{\mathbf{k} \in \Omega} \tilde{v}(\mathbf{k}) \exp (i \mathbf{k r}), \quad \tilde{v}(\mathbf{k})=\int_{V} v(\mathbf{r}) \exp (-i \mathbf{k r}) d^{D} \mathbf{r},
$$

здесь $\tilde{v}(\mathbf{k})$ - фурье-образ потенциала, $D$ - размерность пространства, $\Omega$ - множество всех волновых векторов (область определения функции $\tilde{v}(\mathbf{k}))$.

В соответствии с определением (2) функция $\tilde{v}(\mathbf{k})$ зависит от объема системы $V$. Будем полагать, что межатомный потенциал $v(\mathbf{r})$ при $r=|\mathbf{r}| \rightarrow \infty$ убывает достаточно быстро, чтобы функция $\tilde{v}(\mathbf{k})$, по сушеству, только в термодинамическом пределе $(N, V \rightarrow \infty, N / V=n=\mathrm{const})$ стремяшаяся к фурье-образу потеншиала, не зависела от объема системы. Заметим, что запись $\mathbf{k} \in \Omega$ означает, что суммирование осушествляется не по всем $\mathbf{k}$ из $\Omega$, а лишь по дискретному набору векторов из множества $\Omega$, удовлетворяюших условию квантования

$$
\mathbf{k}=\frac{2 \pi}{L} \mathbf{q}
$$

где $L=V^{1 / D}$ - ребро куба, в котором размещена система частиц, $\mathbf{q}-$ вектор с целочисленными координатами. В соответствии с этими определениями множество $\Omega$ фиксировано, а в процессе термодинамического перехода происходит изменение дискретного набора векторов из этого множества.

Выделим в множестве $\Omega$ три подмножества: $\Omega^{+}, \Omega^{-}, \Omega^{0}$, в которых функция $\tilde{v}(\mathbf{k})$ положительна, отрицательна и равна нулю, соответственно, и определим две строго положительные функции $v^{ \pm}(\mathbf{k})$ :

$$
v^{ \pm}(\mathbf{k})= \begin{cases}\tilde{v}(\mathbf{k}), & \mathbf{k} \in \Omega^{+}, \\ -\tilde{v}(\mathbf{k}), & \mathbf{k} \in \Omega^{-} .\end{cases}
$$

Заметим, что функции $v^{ \pm}(\mathbf{k})$ определены не на $\Omega$, а на подмножествах $\Omega^{ \pm}$, соответственно ${ }^{1)}$.

Выразим теперь полную потенциальную энергию системы частищ через функции $v^{ \pm}(\mathbf{k})$ :

$$
\begin{aligned}
w= & \frac{1}{2} \sum_{\substack{s, s^{\prime}=1 \\
s \neq s^{\prime}}}^{N} v\left(\left|\mathbf{R}_{s}-\mathbf{R}_{s^{\prime}}\right|\right)=\frac{N}{2}(n \tilde{v}(0)-v(0))+\frac{1}{2 V} \sum_{\mathbf{k} \in \Omega^{+}} v^{+}(\mathbf{k})\left[C^{2}(\mathbf{k})+S^{2}(\mathbf{k})\right]- \\
& -\frac{1}{2 V} \sum_{\mathbf{k}^{\prime} \in \Omega^{-}} v^{-}\left(\mathbf{k}^{\prime}\right)\left[C^{2}\left(\mathbf{k}^{\prime}\right)+S^{2}\left(\mathbf{k}^{\prime}\right)\right],
\end{aligned}
$$

\footnotetext{
1) Разбиение функции на положительно- и отрицательно-определенные части неединственно.
} 
где

$$
C\left(\mathbf{k}^{\prime}\right)=\sum_{s=1}^{N} \cos \left(\mathbf{k} \mathbf{R}_{s}\right), \quad S\left(\mathbf{k}^{\prime}\right)=\sum_{s=1}^{N} \sin \left(\mathbf{k} \mathbf{R}_{s}\right),
$$

$N v(0) / 2$ - константа, компенсирующая члены с $s=s^{\prime} ;$ суммы по $\mathbf{k}, \mathbf{k}^{\prime}$ не содержат слагаемого с $\mathbf{k}=0$, оно выделено в виде члена $N^{2} \tilde{v}(0) / 2 V$.

В силу центральности межатомного потенциала из того, что $\mathbf{k} \in \Omega^{+}$, следует $-\mathbf{k} \in \Omega^{+}$(аналогично для $\Omega^{-}$), поэтому набор переменных $C(\mathbf{k}), S(\mathbf{k})$ линейно зависим. Сократим этот набор вдвое, разбив множества $\Omega^{ \pm}$пополам произвольной плоскостью, проходящей через начало координат, и сохраним суммирование только по "половинам" множеств $\Omega^{ \pm} / 2$ в (5) (сохраненный комплект переменных, как нетрудно убедиться, линейно независим). Тогда

$$
\begin{aligned}
w= & \frac{N}{2}(n \tilde{v}(0)-v(0))+\frac{1}{V} \sum_{\mathbf{k} \in \Omega^{+} / 2} v^{+}(\mathbf{k})\left[C^{2}(\mathbf{k})+S^{2}(\mathbf{k})\right]- \\
& -\frac{1}{V} \sum_{\mathbf{k}^{\prime} \in \Omega^{-} / 2} v^{-}\left(\mathbf{k}^{\prime}\right)\left[C^{2}\left(\mathbf{k}^{\prime}\right)+S^{2}\left(\mathbf{k}^{\prime}\right)\right] .
\end{aligned}
$$

Подставив это выражение в конфигурационный интеграл (1), получим произведение по $\mathbf{k}, \mathbf{k}^{\prime}$ экспонент вида

$$
\exp \left\{\frac{\beta v^{+}(\mathbf{k})}{V} C^{2}(\mathbf{k})\right\}, \quad \exp \left\{-\frac{\beta v^{-}\left(\mathbf{k}^{\prime}\right)}{V} C^{2}\left(\mathbf{k}^{\prime}\right)\right\},
$$

каждую из которых представим в виде интегралов Стратоновича-Хаббарда

$$
\begin{aligned}
& \exp \left\{\frac{\beta v^{+}(\mathbf{k})}{V} C^{2}(\mathbf{k})\right\}=\sqrt{\frac{V}{4 \pi \beta v^{+}(\mathbf{k})}} \times \\
& \quad \times \int_{-\infty}^{+\infty} \exp \left[-\frac{V}{4 \beta v^{+}(\mathbf{k})}\left(x^{+}(\mathbf{k})\right)^{2}-i x^{+}(\mathbf{k}) C(\mathbf{k})\right] d x^{+}(\mathbf{k}), \\
& \exp \left\{-\frac{\beta v^{-}\left(\mathbf{k}^{\prime}\right)}{V} C^{2}\left(\mathbf{k}^{\prime}\right)\right\}=\sqrt{\frac{V}{4 \pi \beta v^{-}\left(\mathbf{k}^{\prime}\right)}} \times \\
& \quad \times \int_{-\infty}^{+\infty} \exp \left[-\frac{V}{4 \beta v^{-}\left(\mathbf{k}^{\prime}\right)}\left(x^{-}\left(\mathbf{k}^{\prime}\right)\right)^{2}-x^{-}\left(\mathbf{k}^{\prime}\right) C\left(\mathbf{k}^{\prime}\right)\right] d x^{-}\left(\mathbf{k}^{\prime}\right) .
\end{aligned}
$$

В итоге получим

$$
\begin{aligned}
Z_{\mathrm{c}}= & C \int_{-\infty}^{\infty} \cdots \int_{-\infty}^{\infty}\left(\prod_{\mathbf{k} \in \Omega^{+} / 2} \frac{d x^{+}(\mathbf{k}) d y^{+}(\mathbf{k}) N}{4 \pi n \beta v^{+}(\mathbf{k})}\right)\left(\prod_{\mathbf{k}^{\prime} \in \Omega^{-} / 2} \frac{d x^{-}\left(\mathbf{k}^{\prime}\right) d y^{-}\left(\mathbf{k}^{\prime}\right) N}{4 \pi n \beta v^{-}\left(\mathbf{k}^{\prime}\right)}\right) \times \\
& \times \exp \left[-N G\left(\ldots, x^{+}(\mathbf{k}), y^{+}(\mathbf{k}), \ldots, x^{-}\left(\mathbf{k}^{\prime}\right), y^{-}\left(\mathbf{k}^{\prime}\right), \ldots\right)\right]
\end{aligned}
$$


где

$$
\begin{gathered}
G\left(\ldots, x^{+}(\mathbf{k}), y^{+}(\mathbf{k}), \ldots, x^{-}\left(\mathbf{k}^{\prime}\right), y^{-}\left(\mathbf{k}^{\prime}\right), \ldots\right)=G_{0}-\ln F_{1}= \\
=\sum_{\mathbf{k} \in \Omega^{+} / 2} \frac{\left[\left(x^{+}(\mathbf{k})\right)^{2}+\left(y^{+}(\mathbf{k})\right)^{2}\right]}{4 n \beta v^{+}(\mathbf{k})}+ \\
\quad+\sum_{\mathbf{k}^{\prime} \in \Omega^{-} / 2} \frac{\left[\left(x^{-}\left(\mathbf{k}^{\prime}\right)\right)^{2}+\left(y^{-}\left(\mathbf{k}^{\prime}\right)\right)^{2}\right]}{4 n \beta v^{-}\left(\mathbf{k}^{\prime}\right)}-\ln F_{1}, \\
F_{1}=\int_{V} \frac{d^{D} \mathbf{R}}{V} \exp \left[-i \sum_{\mathbf{k} \in \Omega^{+} / 2}\left(x^{+}(\mathbf{k}) \cos (\mathbf{k R})+y^{+}(\mathbf{k}) \sin (\mathbf{k R})\right)\right] \times \\
\quad \times \exp \left[-\sum_{\mathbf{k}^{\prime} \in \Omega^{-} / 2}\left(x^{-}\left(\mathbf{k}^{\prime}\right) \cos \left(\mathbf{k}^{\prime} \mathbf{R}\right)+y^{-}\left(\mathbf{k}^{\prime}\right) \sin \left(\mathbf{k}^{\prime} \mathbf{R}\right)\right)\right],
\end{gathered}
$$

$x^{ \pm}(\mathbf{k})$ и $y^{ \pm}(\mathbf{k})$ - дополнительные переменные преобразования Стратоновича-Хаббарда,

$$
C=\exp \left\{\frac{N \beta}{2}[v(0)-n \tilde{v}(0)]\right\},
$$

$\beta=1 / T, T$-температура.

В точной формуле (9) подынтегральное выражение для конфигурационного интеграла $Z_{\mathrm{c}}$ “факторизовано” по атомным координатам, поэтому проблемы расцепления атомных координат при интегрировании не возникает. Вместо этого появляется проблема вычисления интеграла универсального вида $F_{1}(11)$, которьй ${ }^{2)}$ представляет собой среднее значение экспоненты, содержашей переменные $x^{ \pm}(\mathbf{k}), y^{ \pm}(\mathbf{k})$ в качестве параметров. В случае компактности носителя функции $\tilde{v}(\mathbf{k})\left(\operatorname{supp} \tilde{v}(\mathbf{k})=\Omega^{+} \cup \Omega^{-}\right)$множество этих переменных до перехода к термодинамическому пределу конечно, но становится бесконечным в этом пределе. В случае некомпактности supp $\tilde{v}(\mathbf{k})$ множество указанных переменных бесконечно даже до перехода к термодинамическому пределу.

Интеграл (9) представляет собой негауссов континуальный интеграл по вспомогательным полям $x^{ \pm}(\mathbf{k}), y^{ \pm}(\mathbf{k})$, которьй дает среднее значение от $N$-й степени функционала $F_{1}(11)$ по гауссовой мере

$$
\begin{aligned}
d \mu= & \left(\prod_{\mathbf{k} \in \Omega^{+} / 2} \frac{d x^{+}(\mathbf{k}) d y^{+}(\mathbf{k}) N}{4 \pi n \beta v^{+}(\mathbf{k})}\right)\left(\prod_{\mathbf{k}^{\prime} \in \Omega^{-} / 2} \frac{d x^{-}\left(\mathbf{k}^{\prime}\right) d y^{-}\left(\mathbf{k}^{\prime}\right) N}{4 \pi n \beta v^{-}\left(\mathbf{k}^{\prime}\right)}\right) \times \\
& \times \exp \left[-N \sum_{\mathbf{k} \in \Omega^{+} / 2} \frac{\left[\left(x^{+}(\mathbf{k})\right)^{2}+\left(y^{+}(\mathbf{k})\right)^{2}\right]}{4 n \beta v^{+}(\mathbf{k})}-\right. \\
& \left.-N \sum_{\mathbf{k}^{\prime} \in \Omega^{-} / 2} \frac{\left[\left(x^{-}\left(\mathbf{k}^{\prime}\right)\right)^{2}+\left(y^{-}\left(\mathbf{k}^{\prime}\right)\right)^{2}\right]}{4 n \beta v^{-}\left(\mathbf{k}^{\prime}\right)}\right]
\end{aligned}
$$

\footnotetext{
2) Универсальность вида интеграла $F_{1}$ означает, что он не зависит от межатомных потенциалов.
} 


\section{3. ДОСТАТОЧНОЕ УСЛОВИЕ СПОНТАННОГО НАРУШЕНИЯ СИММЕТРИИ}

Интересно отметить, что множества $\Omega^{+}$и $\Omega^{-}$дают принципиально отличающиеся вклады в функционал $F_{1}$. Это проявляется уже в довольно грубой оценке

$$
\left|F_{1}\right| \leqslant \exp \left\{\sum_{\mathbf{k}^{\prime} \in \Omega^{-} / 2}\left[\left|x^{-}\left(\mathbf{k}^{\prime}\right)\right|+\left|y^{-}\left(\mathbf{k}^{\prime}\right)\right|\right]\right\} .
$$

Основной вклад в интеграл (9) по мере $\mu$ дают значения $x^{ \pm}(\mathbf{k}), y^{ \pm}(\mathbf{k})$ порядка

$$
\left|x^{ \pm}(\mathbf{k})\right| \simeq\left(\frac{4 \pi \beta v^{ \pm}(\mathbf{k})}{N}\right)^{1 / 2}, \quad\left|y^{ \pm}(\mathbf{k})\right| \simeq\left(\frac{4 \pi \beta v^{ \pm}(\mathbf{k})}{N}\right)^{1 / 2}
$$

т.е. бесконечно малые в термодинамическом пределе. Поэтому целесообразно подробно исследовать окрестность точки $x^{ \pm}(\mathbf{k})=0, y^{ \pm}(\mathbf{k})=0$.

Разлагая $\ln F_{1}$ по $x^{ \pm}(\mathbf{k}), y^{ \pm}(\mathbf{k})$ с точностью до квадратичных членов, найдем в соответствуюшем приближении

$$
\begin{aligned}
G \simeq & \frac{1}{4} \sum_{\mathbf{k} \in \Omega^{+} / 2}\left(\left(x^{+}(\mathbf{k})\right)^{2}+\left(y^{+}(\mathbf{k})\right)^{2}\right)\left[\frac{1}{n \beta v^{+}(\mathbf{k})}+1\right]+ \\
& +\frac{1}{4} \sum_{\mathbf{k}^{\prime} \in \Omega^{-} / 2}\left(\left(x^{-}\left(\mathbf{k}^{\prime}\right)\right)^{2}+\left(y^{-}\left(\mathbf{k}^{\prime}\right)\right)^{2}\right)\left[\frac{1}{n \beta v^{-}\left(\mathbf{k}^{\prime}\right)}-1\right] .
\end{aligned}
$$

Отсюда видно, что при достаточно высоких температурах

$$
\left(n \beta \max _{\mathbf{k} \in \Omega}|\tilde{v}(\mathbf{k})| \ll 1\right)
$$

слагаемыми \pm 1 в квадратных скобках (16) можно пренебречь и мы получим простой случай идеального газа $Z_{\mathrm{c}}=1$. При промежуточных температурах

$$
\left(n \beta \max _{\mathbf{k} \in \Omega}|\tilde{v}(\mathbf{k})|<1\right)
$$

межатомные взаимодействия дают более или менее заметный вклад в интеграл $Z_{\text {c }}$.

Интересное явление имеет место в случае непустого множества $\Omega^{-} \neq \varnothing$, когда при $T=T_{\mathrm{S}}:$

$$
T_{\mathrm{S}}=n \max _{\mathbf{k}^{\prime} \in \Omega^{-}} v^{-}\left(\mathbf{k}^{\prime}\right),
$$

квадратичная форма (16) по полевым переменным $x^{ \pm}(\mathbf{k}), y^{ \pm}(\mathbf{k})$ становится знакопеременной. Точка минимума $x^{ \pm}(\mathbf{k}), y^{ \pm}(\mathbf{k})$ функционала $G$ при $T<T_{\mathrm{s}}$ становится седловой точкой. В этом случае приближение (16) для функции $G$ будет неудовлетворительным, поскольку в гауссовом приближении функциональный интеграл (9) расходится по тем переменным $x^{-}(\mathbf{k}), y^{-}(\mathbf{k})$, для которых $n \beta v^{-}(\mathbf{k}) \geqslant 1$. Строго говоря, эта расходимость 
имеет место только в гауссовом приближении и устраняется при учете членов четвертого порядка по $x^{-}(\mathbf{k}), y^{-}(\mathbf{k})$ в разложении $G$.

С другой стороны, функция $G$ в квадратичном приближении определяет некоторый эффективный лагранжиан, соответствуюший свободным “элементарным возбуждениям". Расходимость интегралов в этом приближении при $T<T_{\mathrm{s}}$ может быть интерпретирована как нестабильность этих элементарных возбуждений [2], а необходимые члены высших порядков приводят к интенсивному взаимодействию элементарных возбуждений и как следствие к перестройке системы. Заметим еще, что более прозрачная интерпретация происходящей перестройки системы может быть получена после вычисления корреляционных функций. Таким образом, при условии

$$
\Omega^{-} \neq \varnothing
$$

сушествует температура $T_{\mathrm{S}}(17)$, ниже которой в системе происходит перестройка, т.е. имеет место спонтанное нарушение симметрии.

Вероятно, впервые температура $T_{\mathrm{s}}$ появилась в работах Власова [3] как точка ветвления решений нелинейного интегрального уравнения для плотности частиц. "Ответвившееся" решение было периодическим в пространстве, и потому точка $T_{\mathrm{S}}$ отождествлялась с температурой кристаллизации. Неудовлетворительность этой интерпретации отмечалась в работе [4]; некоторое обобшение результатов Власова было вьполнено Тябликовым [5], Базаровым $[6,7]$ и другими. Большая часть возражений против подобного описания кристаллизации, высказанных в работе [4], последующими исследованиями [5-7] так и не снята.

Отметим, что нет вполне убедительных оснований отождествлять $T_{\mathrm{S}}$ с температурой фазового перехода, поскольку при приближении $T$ к $T_{\mathrm{S}}$ сверху часть коэффициентов при $x^{ \pm}(\mathbf{k}), y^{ \pm}(\mathbf{k})$ стремится к нулю, из-за чего в разложении функции $G$ по полевым переменным нельзя уже ограничиваться квадратичными слагаемыми, как это сделано в формуле (16). Соответственно интеграл (9) за пределами квадратичного приближения перестает быть гауссовым и в общем случае явно не вычисляется. Описание фазового перехода за пределами приближения (16) с помошью эргодического приближения выполнено в работе [8].

\section{4. МОДЕЛЬНЫЕ ПОТЕНЦИАЛЫ С НЕОТРИЦАТЕЛЬНЫМИ ФУРЬЕ-ГАРМОНИКАМИ}

Рассмотрим случай, когда условие спонтанного нарушения симметрии (18) не выполняется, т.е. рассмотрим модель, в которой отсутствует межатомное притяжение в k-пространстве. В этом случае при вычислении континуального интеграла (9) можно ограничиться квадратичным приближением (16) для $G$ и вычислить получаюшийся гауссов интеграл. В итоге найдем

$$
\ln Z_{\mathrm{c}}=\ln C-\frac{V}{2} \int_{\Omega^{+}} \frac{d^{D} \mathbf{k}}{(2 \pi)^{D}} \ln \left(1+n \beta v^{+}(\mathbf{k})\right)
$$


Поскольку $\Omega^{-}=\varnothing$, а множество $\Omega^{0}$ вклада в интеграл (19) не дает, то функция $v^{+}(\mathbf{k})$ заменяется на функцию $\tilde{v}(\mathbf{k})$, а $\Omega^{+}-$на $\Omega$. После этих замен получим

$$
\ln Z_{\mathrm{c}}=\ln C-\frac{V}{2} \int_{\Omega} \frac{d^{D} \mathbf{k}}{(2 \pi)^{D}} \ln (1+n \beta \tilde{v}(\mathbf{k})) .
$$

Эта формула была получена Зубаревым [9].

Впоследствии идеи Зубарева получили некоторое продолжение в работах $[10,11]$. K сожалению, в этих работах были сделаны недопустимые аппроксимации для фурьеобразов межатомных потенциалов, приводящие к неконтролируемым изменениям потенциалов в реальном пространстве (как известно, интегральный оператор Фурье не ограничен). В результате произошло неявное изменение рассматриваемой модели - короткодействующие в координатном пространстве потенциалы заменены на дальнодействуюшие. В связи с этим остается неясным, какая часть результатов, содержашихся в указанных работах, соответствует исходным моделям (в частности, используемой авторами модели Изинга), а какие результаты появляются вследствие приближенных математических преобразований.

В этих работах не было замечено, что в системе может иметь место фазовый переход и в отсутствие притяжения в $\mathbf{k - п р о с т р а н с т в е . ~ О б с у д и м ~ э т о т ~ в о п р о с ~ п о д р о б н е е . ~ П о л о ж и м , ~}$ что фурье-образ потенциала неотрицателен, $\tilde{v}(\mathbf{k}) \geqslant 0$, это соответствует отталкиванию в $\mathbf{k}$-пространстве. Заметим, что к этому классу относятся многие интересные потенциалы. К примеру, обобщенный потенциал Морзе, суперпозиция двух потенциалов Юкавы при определенном выборе коэффициентов и некоторые другие имеют неотрицательные фурье-образы и при этом вьглядят вполне "реалистично" в координатном пространстве: с отталкиванием на малых расстояниях, притяжением на больших расстояниях и минимумом на определенном расстоянии. Заметим, что подынтегральное выражение в формуле (20) не содержит никаких особенностей на вешественной температурной оси. Тем не менее фазовый переход оказывается возможным вследствие нарушения условия устойчивости.

Из выражения (20) найдем давление

$$
P=P_{\text {ид }}+\frac{n^{2} \tilde{v}(0)}{2}-\frac{T}{2} \int_{\Omega} \frac{d^{D} \mathbf{k}}{(2 \pi)^{D}}\left[\ln (1+n \beta \tilde{v}(\mathbf{k}))-\frac{n \beta \tilde{v}(\mathbf{k})}{1+n \beta \tilde{v}(\mathbf{k})}\right],
$$

где $P_{\text {ид }}=n T$ - давление идеального газа.

Условие устойчивости по отношению к флуктуациям плотности имеет вид

$$
\frac{\partial P}{\partial n}>0
$$

Интерпретация этого условия состоит в следуюшем. Если в некотором интервале плотностей $n$ функция $P(n)$ монотонно возрастает, то в областях пространства с большей плотностью локальное давление также больше и происходит выравнивание плотностей. Если же функция $P(n)$ на некотором промежутке плотностей убывает, то в областях 
пространства с большей плотностью локальное давление меньше, вследствие чего неизбежные флуктуации локальной плотности начинают расти. Таким образом, достаточным условием фазового перехода в системе является разрешимость системы уравнений

$$
\begin{gathered}
\frac{\partial P}{\partial n}=T+n \tilde{v}(0)-\frac{T}{2} \int_{\Omega} \frac{d^{D} \mathbf{k}}{(2 \pi)^{D}} \frac{n(\beta \tilde{v}(\mathbf{k}))^{2}}{(1+n \beta \tilde{v}(\mathbf{k}))^{2}}=0 \\
\frac{\partial^{2} P}{\partial n^{2}}=\tilde{v}(0)-\frac{T}{2} \int_{\Omega} \frac{d^{D} \mathbf{k}}{(2 \pi)^{D}} \frac{(\beta \tilde{v}(\mathbf{k}))^{2}(1-n \beta \tilde{v}(\mathbf{k}))}{(1+n \beta \tilde{v}(\mathbf{k}))^{3}}=0
\end{gathered}
$$

вместе с выражением (21) в области положительных $n_{0}, P_{0}, \beta_{0}$.

В обшем случае произвольного потенциала $\tilde{v}(\mathbf{k})$ получение аналитического решения системы уравнений (23) не представляется возможным. Рассмотрим в качестве примера трехмерный потенциал Юкавы, фурье-образ которого имеет вид

$$
\tilde{v}(\mathbf{k})=\frac{A}{k^{2}+a^{2}}
$$

После интегрирования по $\mathbf{k}$ найдем уравнение состояния

$$
P=n T+\frac{n^{2} \tilde{v}(0)}{2}-\frac{T a^{3}}{12 \pi}\left[1-\sqrt{1+n \beta \tilde{v}(0)}\left(1-\frac{n \beta \tilde{v}(0)}{2}\right)\right]
$$

Подставляя уравнение состояния в систему (23) и решая ее, получим

$$
n_{0}=\frac{a^{3}}{12 \pi \sqrt{3}}, \quad \beta_{0}=\frac{24 \pi \sqrt{3}}{a^{3} \tilde{v}(0)} .
$$

Отметим, что критическая сжимаемость $\psi=P_{0} / n_{0} T_{0}$ для системы с потенциалом Юкавы равна $2-\sqrt{3} \approx 0.268$; это значительно лучше соответствует экспериментальным значениям, чем решеточные модели работ $[12,13]$ или модель Ван-дер-Ваальса.

Уравнение состояния (25) приводится к безразмерной форме

$$
\pi(\tau, \omega)=\frac{1}{\psi}\left(\tau \omega+\omega^{2}-\sqrt{3}\left[1-\sqrt{1+\frac{2 \omega}{\tau}}\left(1-\frac{\omega}{\tau}\right)\right]\right)
$$

где $\tau=T / T_{0}, \omega=V_{0} / V=n / n_{0}, \pi=P / P_{0}$ - приведенные температура, плотность и давление, соответственно.

Изотермы, построенные с помошью уравнения (27), представляют собой непрерывные кривые, имеюшие при $0<\tau<1$ характерные “ван-дер-ваальсовы” петли и вертикальную асимптоту $1 / \omega=0$, как это и должно быть для потенциала взаимодействия без твердой сердшевины (ван-дер-ваальсовы изотермы, как известно, имеют вертикальную асимптоту $1 / \omega=1 / 3)$. 
Граница метастабильности системы, т.е. устойчивости по отношению к бесконечно малым флуктуациям локальной плотности, называется спинодалью ${ }^{3)}$. Получим уравнение спинодали системы частиц, взаимодействующих между собой через потенциал Юкавы (в безразмерной форме). Граница метастабильности системы определяется условием

$$
\frac{\partial \pi}{\partial \omega}=0
$$

отсюда для юкавской системы имеем

$$
\left(1+\frac{2 \omega}{\tau}\right)^{3 / 2}=\frac{3 \sqrt{3}}{\tau^{2}} \frac{\omega}{\tau}
$$

Элементарный анализ показывает, что при $\tau>1$, т.е. при температурах выше критической, это уравнение относительно $2 \omega / \tau$ не имеет положительных корней. При $\tau=1$ имеется двукратный корень $\omega / \tau=1$, а при $\tau<1$ - два положительных корня, которые без труда могут быть найдены.

Нетрудно показать также, что в критической точке $(\tau=1, \omega=1)$ изотермическая сжимаемость бесконечна, а для всех докритических изотерм $(\tau<1)$ существует область нестабильности, в которой $\partial \pi / \partial \omega>0$.

\section{5. ЗАКЛЮЧЕНИЕ}

Возможность точного решения системы уравнений (23) не ограничивается рассмотренным трехмерным потенциалом Юкавы. В частности, эта система может быть решена точно с фурье-образом вида (24) при размерностях пространства $D=1,2$, а с фурье-образом вида

$$
\tilde{v}(\mathbf{k})=\frac{A}{|\mathbf{k}|^{4}+a^{4}}
$$

- при размерностях пространства $D=1,2,3$. Во всех перечисленных случаях также имеет место фазовый переход.

Отметим еще, что при необходимости рассмотрения более сложных модельных потенциалов (к примеру, потенциала Морзе или суперпозиций потенциалов Юкавы) не представляет особого труда численное решение системы уравнений (23). Соответственно имеется возможность анализа и согласования с экспериментальными данными потенциалов с неотрицательными фурье-образами "реалистического" вида.

Благодарности. Считаем своим приятным долгом поблагодарить рецензента журнала за полезные замечания, способствовавшие улучшению окончательного варианта статьи.

\footnotetext{
3) В случае фазового перехода газ-жидкость точки спинодали определяют границы возможных перегрева жидкости и переохлаждения газа.
} 


\section{Список литературы}

[1] A. Yu. Zakharov. Phys. Lett. A. 1990. V. 147. № 8/9. P. 442.

[2] D. J. Amit. Field Theory, the Renormalization Group, and Critical Phenomena. New York: McGraw-Hill, 1978.

[3] А.А. Власов. Теория многих частиц. М.: ГИТТЛ, 1950.

[4] В. Л. Гинзбург, Л. Д. Ландау, М. А. Леонтович, В. А. Фок. ЖЭТФ. 1946. Т. 16. № 3. C. 246.

[5] С. В. Тябликов. ЖЭТФ. 1947. Т. 17. № 5. С. 386.

[6] И. П. Базаров. Статистическая теория кристаллического состояния. М.: МГУ, 1972.

[7] И. П. Базаров, Э. В. Геворкян. Статистическая теория твердых и жидких кристаллов. М.: МГУ, 1983.

[8] А.Ю. Захаров, И. К. Локтионов, Я.И. Грановский. ТВТ. 1997. Т. 35. № 3. С. 367.

[9] Д. Н. Зубарев. ДАН СССР. 1954. Т. 95. № 4. С. 757.

[10] И.Р. Юхновский, М. Ф. Головко. Статистическая теория равновесных классических систем. Киев: Наукова думка, 1980.

[11] И. Р. Юхновский. Теория фазовых переходов второго рода. Метод коллективных переменных. Киев: Наукова думка, 1985.

[12] А.Ю. Захаров, С. В. Терехов. Обобщенная решеточная модель фазовых равновесий в многокомпонентных системах. В сб.: Математические задачи химической термодинамики. Ред. Г. А. Коковин. Новосибирск: Наука, 1985.

[13] Г. Стенли. Фазовые переходы и критические явления. М.: Мир, 1973.

Поступила в редакцию 26.III.1998 г., после доработки 3.IX.1998 г. 